\title{
LA PEDOANTRACOLOGÍA EN ESPAÑA: UNA REVISIÓN BIBLIOGRÁFICA
}

\author{
Pedoanthracology in Spain: a bibliographic review \\ Rubén Pardo Martínez \\ Departamento de Análisis Geográfico Regional y Geografía Física de la Universidad de Granada \\ Identificador ORCID de los autores y e-mail \\ Rubén Pardo Martínez: https://orcid.org/0000-0003-4457-8536. Email: rubenpardo@ugr.es
}

\begin{abstract}
Recibido: 12-05-2020. Aceptado: 31-07-2020. Fecha de publicación on-line: 23-09-2020
Citation / Cómo citar este artículo: Pardo Martínez, R. (2020). La pedoantracología en España: una revisión bibliográfica. Pirineos, 175, e059. https://doi.org/10.3989/pirineos.2020.175009
\end{abstract}

RESUMEN: La pedoantracología se define como la búsqueda, tratamiento y análisis taxonómico de carbón procedente de vegetación leñosa incendiada en suelos no arqueológicos. Sus aplicaciones han permitido cubrir un amplio espectro de temáticas, sobre todo cuando ha formado parte de aproximaciones paleoecológicas multidisciplinares. En líneas generales, esta metodología ha manifestado un interés creciente dentro de las disciplinas paleoecológicas, aunque actualmente existen enormes diferencias territoriales, de manera que Francia -territorio pionero en la aplicación de esta metodologíaproduce buena parte de las publicaciones pedoantracológicas europeas. En España, su aplicación en Pirineos, Sistema Central, Cordillera Bética y Cordillera Cantábrica ha sido relativamente reciente, no conociéndose en consecuencia ni su trayectoria ni su estado actual. Por ello, en este trabajo se presenta un análisis detallado y comparativo de todas aquellas investigaciones de carácter pedoantracológico llevadas a cabo en este último país, lo que ha permitido conocer cuáles han sido las conclusiones más relevantes obtenidas por los diferentes grupos de trabajo. Este análisis ha puesto de manifiesto que se trata de una metodología que ha obtenido resultados de gran interés a la hora de resolver debates fitosociológicos clásicos, reconstruir antiguas áreas de distribución de especies o determinar cambios en el límite superior del bosque, resultados que han contribuido y pueden seguir contribuyendo al desarrollo de una gestión forestal sostenible más eficaz. Por su parte, el futuro de la disciplina muestra un progresivo avance hacia otros sistemas montañosos, constatando que se trata de una metodología que ha sabido ganarse su lugar dentro de las disciplinas paleoecológicas.

PALABRAS CLAVE: Carbones; suelos naturales; revisión bibliográfica; paleoecología; España.

ABSTRACT: Pedoanthracology is defined as the search, treatment and taxonomic analysis of charcoal from burned woody vegetation in non-archaelogical soils. Its applications have allowed studying a wide spectrum of topics, especially when it has been part of multidisciplinary paleoecological approaches. In general terms, this methodology has shown growing interest within paleoecological disciplines, although there are currently enormous territorial differences, such that France -a pioneer territory in the application of this methodology-is the country where a large part of pedoanthracological publications in Europe are written. In others, such as Spain, its application in Pyrenees, Central System, Baetic 


\section{• R. PARDO MARTÍNEZ}

System and Cantabrian Range has been relatively recent, neither its trajectory nor its current status being known. For this reason, this work presents a detailed and comparative analysis of all those investigations of a pedoanthracological nature carried out, which has allowed us to know what the most relevant conclusions obtained by the different working groups have been. This analysis has shown that it is a methodology that has obtained results of great interest when solving classical phytosociological debates, reconstructing old species distribution areas or determining changes in the treeline, results that can contribute and can continue contributing to development of more effective sustainable forest management. Also, the future of the discipline shows a progressive advance towards others mountain systems, confirming that it is a methodology that has managed to earn its place within paleoecological disciplines.

KEY WORDS: Charcoals; natural soils; bibliographic review; paleoecology; Spain.

\section{Introducción}

La caracterización y dinámica paleobiogeográfica de la vegetación actual constituye un pilar fundamental a la hora de comprender los principios explicativos que ayudan a interpretar las relaciones entre especies y ambientes. En la naturaleza, muchos patrones no pueden ser entendidos si no se analizan bajo una escala temporal amplia -de cientos a miles de años-(Willis \& Birks, 2006). Es por ello que los estudios de corte paleoecológico ofrecen una magnífica oportunidad para predecir los posibles comportamientos de la vegetación ante escenarios dinámicos de Cambio Global, constituyendo una herramienta de vital importancia a la hora de orientar las estrategias de gestión y conservación sostenible en espacios naturales (Willis et al., 2007; Saulnier-Talbot, 2016).

Las posibilidades a la hora de reconstruir ambientes y condiciones ecológicas pretéritas son múltiples, disponiendo de un extenso catálogo de bioindicadores y señales paleoambientales que, bajo unas condiciones específicas de conservación, son estudiadas por diferentes disciplinas paleocológicas. Algunas de ellas, como la palinología, poseen una larga tradición científica en buena parte de los países desarrollados, que en el caso de España se inicia con los trabajos de Bellot \& Vieitez (1945). En otros casos su desarrollo es mucho más reducido, tal y como sucede con la pedoantracología, disciplina que se encarga del estudio de carbón procedente de vegetación leñosa en suelos no arqueológicos para su cuantificación -o cálculo de antracomasa- y su identificación taxonómica (Thinon, 1992), con el objeto de estudiar la composición de comunidades de plantas leñosas del pasado a una escala muy detallada, procedimientos que normalmente van acompañados de dataciones radiocarbónicas (Nelle et al., 2013).

Este carbón es una de las consecuencias directas de los recurrentes incendios -ya sean naturales o de origen antrópico- que se han sucedido a lo largo del Holoceno. $\mathrm{Su}$ estudio se convierte en un excepcional recurso complementario para cualquier aproximación paleoecológica y paleobiogeográfica a un ámbito espacial concreto, siendo de gran utilidad como fuente para el conocimiento geográfico de los incendios forestales -análisis de microcarbones- (Turner et al., 2008), así como para reconstruir con gran precisión espacial la dinámica de la vegetación en el pasado (Carcaillet \& Talon, 1996; Talon et al., 1998). De hecho, el fuego constituye uno de los principales agentes modeladores del paisaje vegetal a lo largo de la historia, siendo un elemento clave para explicar la gran diversidad de especies asociadas al clima mediterráneo (Bond \& Keeley, 2005).

Bajo estas consideraciones, primero la antracología, cuyo objeto de estudio es tanto el carbón resultante de una mala combustión como aquel que ha sido transformado voluntariamente, iniciaría su andandura en el panorama científico a partir de la investigación de Unger (1846). En este apartado se incluyen los restos presentes en suelos geológicos y en yacimientos arqueológicos, con especial atención a estos últimos, donde la acción antrópica en hogares, hornos, carboneras, etc. jugó un papel fundamental en la génesis de estos registros paleoecológicos (Uzquiano, 1997). Posteriormente, la pedoantracología, desarrollada por Thino (1978), centraría el foco de atención en los carbones procedentes de paleosuelos -fuera de contextos arqueológicos-. A este respecto se considera toda aquella madera carbonizada que se origina como respuesta a incendios de origen natural y antrópico, conservándose como un registro no estratigráfico, lo que obliga a datar necesariamente cada una de las muestras (Thinon, 1992).

En consecuencia, ambas disciplinas posibilitan la reconstrucción de la historia ecológica de especies y/o comunidades. El proceder de una y otra comparte similitudes evidentes en cuanto a la búsqueda, triado e identificación del carbón, si bien es en el tamaño de éste donde ambas metodologías encuentran sus mayores desemejanzas. La práctica totalidad de ensayos pedoantracológicos trabaja fracciones de madera carbonizada con dimensiones que generalmente suelen oscilar entre 5 y $1 \mathrm{~mm}$-pasos de malla de 5, 2 y $0,8 \mathrm{~mm}$ (Nelle et al., 2013), mientras que el método antracológico, salvo que haya resultados significativos, deshecha los fragmentos más finos (Oliva-Rodríguez Ariza, 2006).

Tanto la aplicabilidad como el interés de la pedoantracología han ido incrementándose durante las últimas décadas, si bien la extensa bibliografía pedoantracológica se concentra mayoritariamente en Europa y, en particular, en Francia. En otros países como Italia, Alemania o República Checa esta disciplina también ha sido desarrollada durante los últimos años (Nelle et al., 2013). 
En el caso de España, la aplicación del método pedoantracológico es relativamente reciente, iniciándose los trabajos en la cara sur del macizo pirenaico a partir de la tesis doctoral de Cunill (2010). Posteriormente esta metodología paleoecológica se expandiría hacia otros sistemas montañosos de España, como la Cordillera Prelitoral catalana, el Sistema Central, la Cordillera Bética y la Cordillera Cantábrica.

En este contexto metodológico y de conocimiento, este trabajo pretende hacer una revisión bibliográfica de los diferentes ensayos pedoantracológicos realizados hasta el momento en España, llevando a cabo un análisis detallado y comparativo de todas aquellas cuestiones que pudiesen ser de interés desde el punto de vista pedoantracológico y paleoecológico. Ello posibilita, en primer lugar, conocer cuál ha sido la trayectoria de la disciplina en España y su posición relativa dentro del continente europeo, mostrando información relevante sobre las variables estudiadas, líneas de investigación seguidas, principales resultados obtenidos y conclusiones derivadas de los diferentes ensayos realizados. Por otra parte, este análisis permite conocer las principales aplicaciones que ha tenido hasta el momento la pedoantracología como disciplina paleoecológica, y cómo sus resultados pueden sentar las bases de determinadas políticas de gestión forestal en diferentes espacios naturales de España.

\section{Métodos}

\subsection{Búsqueda en base de datos}

En primer lugar, se llevó a cabo una búsqueda sistematizada de todas aquellas investigaciones que integrasen -entre alguna de sus palabras clave o bien dentro del tema, el resumen o el título- conceptos como antracología y/o pedoantracología, lo cual se hizo tanto en castellano como en inglés (anthracology y pedoanthraco$\log y$ ). El propósito de incluir en esta búsqueda preliminar el término "antracología" fue el de poder obtener información que pudiese ser de interés para la investigación, como así fue, por ejemplo, a la hora de contextualizar los antecedentes de este ensayo. Para esta búsqueda se emplearon las principales bases de datos académicas, como Web of Science, Scopus, BASE, Dialnet, Springer Link, Researchgate o Google Schoolar, entre otras.

Una vez completada esta primera búsqueda en bruto, el siguiente paso fue filtrar los resultados según localización, es decir, atendiendo al ámbito de estudio y/o de aplicación de la investigación. Esta depuración permitió que se excluyeran aquellos trabajos referentes a ámbitos fuera de España, de manera acorde a los objetivos de la investigación. En total, se obtuvieron en un primer momento 212 documentos en Web of Sciences, 207 en Scopus o 96 en BASE, por ejemplo, que finalmente quedaron en algunas decenas o unidades (según la base de datos consultada) tras aplicar los filtros anteriormente mencionados.
Seguidamente se procedió a la lectura individualizada de los resúmenes y metodología de cada uno de los documentos obtenidos: un total de 34 (tanto en castellano como en inglés). Con ello se excluyó aquella bibliografía que, o bien trabajaba con carbón desde un punto de vista complementario o parcial (caso de los microcarbones), o lo hacía desde una perspectiva arqueológica, es decir, bajo el prisma de la antracología.

En este punto también se excluyeron aquellos documentos referentes a comunicaciones y pósteres presentados en congresos, ya que éstos son resultados previos de ensayos pedoantracológicos publicados posteriormente en forma de artículo científico.

Seleccionados los documentos estrictamente pedoantracológicos, se procedió a su análisis interpretativo y categorizado atendiendo a criterios como la fecha de publicación, la autoría, el ámbito de estudio, los objetivos, las líneas de aplicabilidad, el idioma y otra serie de datos de carácter pedoantracológico y paleoecológico que pudiesen arrojar información de interés para la presente investigación.

Por último, cabe mencionar que, aunque buena parte de la bibliografía se encontraba disponible en formato digital, en algunos casos -capítulos de libro, principalmente- hubo que recurrir al formato impreso para poder realizar la revisión de todos aquellos datos relevantes para la investigación.

\subsection{Entrevistas informales}

Tras la revisión bibliográfica inicial, se contactó con algunos de los autores de los diferentes estudios pedoantracológicos recopilados. Esto permitió corroborar los resultados de la primera fase, al tiempo que posibilitó conocer las posibles líneas futuras de trabajo en materia de pedoantracología. Las entrevistas en cuestión no tenían una estructura previamente determinada ni categorizada, apostándose por un carácter informal de las mismas con el fin de facilitar el intercambio directo de información entre ambas partes (Vargas-Jiménez, 2012).

Después de abordar tanto la revisión bibliográfica como las entrevistas informales, el resultado final ha sido la consideración de un total de 22 trabajos en los que, de forma explícita o interdisciplinar, la pedoantracología constituye una de las metodologías de trabajo utilizadas para la consecución de los objetivos propuestos.

\section{Resultados}

Los resultados, sintetizados en la tabla 1 , ofrecen la siguiente información:

\subsection{Autoría}

Se ha contabilizado un total de 24 autores y coautores -22 españoles y 2 franceses-, con una participación porcentual que difiere enormemente según casos. De esta manera, 


\section{$4 \cdot$ R. PARDO MARTÍNEZ}

Cunill, del Departamento de Geografía de la Universidad Autónoma de Barcelona (UAB en adelante) y miembro del Grup de Recerca de Àrees de Muntanya i Paisatje (GRAMP en adelante), es el apellido más repetido en los trabajos de pedoantracología a nivel español, participando -bien como autora o coautora- en más del $86 \%$ de las publicaciones.

Tabla 1: Síntesis informativa por áreas de estudio derivada de la bibliografía consultada. Fuente: elaboración propia.

Table 1: Informative synthesis by areas of study derived from the consulted bibliography. Source: own elaboration.

\section{PIRINEOS}

1. Cunill, R., 2010. Estudi interdisciplinari de l'evolució del límit superior del bosc durant el período holocènic a la zona de Plaus de Boldís-Montarenyo, Pirineu central català. Tesis Doctoral, Universidad Autónoma de Barcelona.

2. Cunill-Artigas, R., Pèlachs, A. \& Soriano, J.M., 2008. The consequences on landscape of new land uses in the upper forest line. In: Naturbanization: New identities and processes for rural-natural areas (ed. M.J. Prados). CRC Press/Balkema, Leiden.

3. Cunill-Artigas, R., Soriano, J.M., Bal, M.C., Pèlachs, A. \& Pérez-Obiol, R., 2012. Holocene treeline changes on the south slope of the Pyrenees: a pedoanthracological analysis. Vegetation History and Archaeobotany, 21(4): 373-384. https://doi. org/10.1007/s00334-011-0342-y

4. Cunill-Artigas, R., Soriano, J.M., Bal, M.C., Pèlachs, A., Rodríguez, J.M. \& Pérez-Obiol, R., 2013. Holocene high-altitude vegetation dynamics in the Pyrenees: a pedoanthracology contribution to an interdisciplinary approach. Quaternary International, 289: 60-70. https://doi.org/10.1016/j.quaint.2012.04.041

5. Pèlachs, A., Pérez-Obiol, R., Soriano, J.M., Cunill, R., Bal, M.C. \& García-Codron, J.C., 2017. The Role of Environmental Geohistory in High-Mountain Landscape Conservation. In: High Mountain Conservation in a Changing World. Advances in Global Change Research (eds. J. Catalan, J. Ninot \& M. Aniz). Springer, pp. 107-129, Berlin.

6. Soriano, J.M., Pèlachs-Mañosa, A., Pérez-Obiol, R., Rodríguez, J.M. \& Cunill-Artigas, R., 2016. Dinámica del abeto en el Pirineo catalán durante los últimos 10.000 años. In: Avances en Biogeografia: áreas de distribución: entre puentes y barreras (eds. J. Gómez, J. Arias, J.A. Olmedo \& J.L. Serrano). Editorial Universidad de Granada-Tundra Ediciones, pp. 544-552, Granada y Castellón.

\begin{tabular}{|l|l|}
\hline Año de publicación & 2008 (2), 2010 (1), 2012 (3), 2013 (4), 2016 (6) y 2017 (5). \\
\hline Tipo de documento & Tesis doctoral (1), capítulos de libro (2, 5 y 6) y artículos científicos (3 y 4). \\
\hline Importancia de la pedoantracología & $\begin{array}{l}\text { Alta: la pedoantracología constituye una de las metodologías de trabajo principales para la } \\
\text { consecución de los objetivos propuestos (1, 2, 3 y 4). } \\
\text { Media: la pedoantracología actúa como fuente paleoecológica complementaria (5 y 6). }\end{array}$ \\
\hline Sondeos pedológicos & $\begin{array}{l}\text { 8 sondeos sobre litologías metamórficas (entre 1.996 y 2.593 m s. n. m.) (1, 3, 4 y 5). } \\
\text { 1 sondeo sobre litologías metamórficas (1.150 m s. n. m.) (6). } \\
\text { No se especifica (2). }\end{array}$ \\
\hline Objetivo/s principal/es & $\begin{array}{l}\text { Estudiar la evolución del límite superior del bosque (1, 2, 3 y 4), contribuir con } \\
\text { información y reflexión sobrelíneas de base de diversidad, umbrales, resiliencia y } \\
\text { restauración de procesos ecológicos (5) y conocer con precisión la distribución de los } \\
\text { abetales en el pasado (6). }\end{array}$ \\
\hline Taxones identificados & $\begin{array}{l}\text { Especies: Abies alba, Genista balansae, Pinus sylvestris/uncinata. } \\
\text { Géneros: Betula. } \\
\text { Familias: ericácea. }\end{array}$ \\
\hline Valores de antracomasa & Entre 0,2 y 1.730 mg/kg. \\
\hline Dataciones radiocarbónicas & $\begin{array}{l}\text { 13 dataciones con cronologías comprendidas entre 679 y 10.796 años antes del presente } \\
\text { (BP en adelante) (1, 3, 4 y 5). } \\
\text { No se realizan (2 y 6). }\end{array}$ \\
\hline Tipo de aproximación & Ecológica (1, 3, 4 y 5), geográfica (6) y no se especifica (2). \\
\hline \multicolumn{1}{|c|}{ PIRINEOS Y CORDILLERA CANTÁBRICA }
\end{tabular}

1. Carracedo-Martín, V., Cunill-Artigas, R., García-Codrón, J.C., Pèlachs-Mañosa, A., Pérez-Obiol, R. \& Soriano-López, J.M., 2017. Fuentes para la geografía histórica de los incendios forestales. Algunas consideraciones metodológicas. Cuadernos Geográficos 56(3): 66-89.

2. García-Codrón, J.C., Pèlachs-Mañosa, A. \& Carracedo-Martín, V., 2016. Fuentes para la historia de los incendios forestales y su impacto en la vegetación: puentes y barreras metodológicas. In: Avances en Biogeografia: áreas de distribución: entre puentes y barreras (eds. J. Gómez, J. Arias, J.A. Olmedo \& J.L. Serrano). Editorial Universidad de Granada-Tundra Ediciones, pp. 563-571, Granada y Castellón.

3. Pèlachs, A., García-Codrón, J.C., Soriano, J.M., Pérez-Obiol, R. \& Catalán, J., 2016. Papel de los incendios en las dinámicas forestales del Norte de la Península Ibérica durante el Holoceno. In: Avances en Biogeografía: áreas de distribución: entre puentes y barreras (eds. J. Gómez, J. Arias, J.A. Olmedo \& J.L. Serrano). Editorial Universidad de Granada-Tundra Ediciones, pp. 553-562, Granada y Castellón. 


\begin{tabular}{|l|l|}
\hline Año de publicación & 2016 (2 y 3) y 2017 (1). \\
\hline Tipo de documento & Artículo científico (1) y capitulo de libro (2 y 3). \\
\hline Importancia de la pedoantracología & Media: la pedoantracología actúa como fuente paleoecológica complementaria (1, 2 y 3). \\
\hline Sondeos pedológicos & No se especifican. \\
\hline Objetivo/s principal/es & Conocer la geohistoria de los incendios y de la cubierta vegetal (1, 2 y 3). \\
\hline Taxones identificados & No se especifican. \\
\hline Valores de antracomasa & No se especifican. \\
\hline Dataciones radiocarbónicas & No se realizan. \\
\hline Tipo de aproximación & No se especifica. \\
\hline
\end{tabular}

\section{CORDILLERA CANTÁBRICA}

1. Beato-Bergua, S., 2018. El patrimonio natural de la Sierra del Aramo (Montaña Central Asturiana) y la evolución de su paisaje. Tesis Doctoral, Universidad de Oviedo.

2. Beato-Bergua, S., 2019. El patrimonio natural de la Sierra del Aramo (Montaña Central Asturiana) y la evolución de su paisaje (Summary of Doctoral dissertation, Universidad de Oviedo, Spain). Boletín de la Asociación de Geógrafos Españoles, 80, 2756, 1-5.

3. Beato-Bergua, S., Poblete-Piedrabuena, M.A. \& Cunill-Artigas, R., 2019. Taxus baccata en la Sierra del Aramo (Macizo Central Asturiano). Boletín de la Asociación de Geógrafos Españoles, 81: 1-30. https://doi.org/10.21138/bage.2772

\begin{tabular}{|l|l|}
\hline Año de publicación & 2018 (1) y 2019 (2 y 3). \\
\hline Tipo de documento & Tesis doctoral (1) y artículos científicos (2 y 3). \\
\hline Importancia de la pedoantracología & Media: la pedoantracología actúa como fuente paleoecológica complementaria. \\
\hline Sondeos pedológicos & 2 sondeos sobre litologías sedimentarias (entre 1.611 y 1.701 m s. n. m.). \\
\hline Objetivo/s principal/es & Aumentar el conocimiento biogeográfico sobre el tejo. \\
\hline Taxones identificados & $\begin{array}{l}\text { Géneros: Betula, Corylus, Fagus, Ilex, Juniperus, Quercus, Taxus. } \\
\text { Familias: ericácea. }\end{array}$ \\
\hline Valores de antracomasa & Entre 23,75 y 28,77 mg/kg. \\
\hline Dataciones radiocarbónicas & $\begin{array}{l}7 \text { dataciones radiocarbónicas con cronologías comprendidas entre edades subactuales y } \\
3.450 \text { BP. }\end{array}$ \\
\hline Tipo de aproximación & Geográfica. \\
\hline
\end{tabular}

\section{CORDILLERA PRELITORAL CATALANA}

1. Cunill-Artigas, R., Pèlachs-Mañosa, A., Soriano, J.M., Pérez-Obiol, R., Nadal-Tersa, J. \& Sánchez-Morales, M., 2018. Evolución paleobiogeográfica del abeto (Abies alba) en su límite meridional ibérico (Parc Natural del Montseny, Girona-Barcelona). In: Bosque mediterráneo y humedales: paisaje, evolución y conservación. Aportaciones desde la Biogeografía (eds. R.U. Gosálbez, M.C. Díaz, J.L. García, M.A. Serrano de la Cruz \& O. Jerez). Almud, Ediciones de Castilla-La Mancha, pp. 860-868, Ciudad Real.

\begin{tabular}{|l|l|}
\hline Año de publicación & 2018. \\
\hline Tipo de documento & Capítulo de libro. \\
\hline Importancia de la pedoantracología & $\begin{array}{l}\text { Alta: la pedoantracología constituye una de las metodologías de trabajo principales para la } \\
\text { consecución de los objetivos propuestos. }\end{array}$ \\
\hline Sondeos pedológicos & 3 sondeos sobre litologías metamórficas (entre 1.357 y 1.605 m s. n. m.). \\
\hline Objetivo/s principal/es & Conocer la evolución paleobiogeográfica del abeto. \\
\hline Taxones identificados & $\begin{array}{l}\text { Géneros: Abies, Betula, Calluna, Corylus Daphne, Erica, Fagus, Juniperus, Pinus, Prunus, } \\
\text { Fuercus. } \\
\text { Familias: ericácea, fabácea, rosácea. }\end{array}$ \\
\hline Valores de antracomasa & No se especifica. \\
\hline Dataciones radiocarbónicas & 6 dataciones con cronologías comprendidas entre edades subactuales y 4.095 BP. \\
\hline Tipo de aproximación & Geográfica. \\
\hline
\end{tabular}




\section{SISTEMA CENTRAL}

1. García-Álvarez, S., Bal, M.C., Allée, P., García-Amorena, I. \& Rubiales, J.M., 2017. Holocene treeline history of a highmountain landscape inferred from soil charcoal: The case of Sierra de Gredos (Iberian Central System, SW Europe). Quaternary International, 457(1): 85-98. https://doi.org/10.1016/j.quaint.2017.04.019

\begin{tabular}{|l|l|}
\hline Año de publicación & 2017. \\
\hline Tipo de documento & Artículo científico. \\
\hline Importancia de la pedoantracología & $\begin{array}{l}\text { Alta: la pedoantracología constituye una de las metodologías de trabajo principales para la } \\
\text { consecución de los objetivos propuestos. }\end{array}$ \\
\hline Sondeos pedológicos & 6 sondeos sobre materiales metamórficos égneos (entre 1.700 y $2.200 \mathrm{~m}$ s. n. m.). \\
\hline Objetivo/s principal/es & Reconstruir el límite altitudinal del bosque. \\
\hline Taxones identificados & $\begin{array}{l}\text { Géneros: Betula, Pinus, Populus, Quercus. } \\
\text { Familias: ericácea, leguminosa, rosácea, saliácea. }\end{array}$ \\
\hline Valores de antracomasa & Entre 1.373 y 46.868 mg/kg. \\
\hline Dataciones radiocarbónicas & 23 dataciones radiocarbónicas con cronologías comprendidas entre 1.102 y 8.914 BP. \\
\hline Tipo de aproximación & Ecológica. \\
\hline
\end{tabular}

\section{CORDILLERA BÉTICA}

1. Gómez-Zotano, J., Olmedo-Cobo, J.A., Martínez-Ibarra, E. \& Cunill-Artigas, R., 2015. Descubrimiento y caracterización de una cuenca endorreica en la cumbre de Sierra Bermeja (provincia de Málaga). En Análisis espacial y representación geográfica: innovación y aplicación (eds. J. de la Riva, P. Ibarra, R. Montorio \& M. Rodrigues). Universidad de ZaragozaAGE, pp. 1629-1638, Zaragoza.

2. Gómez-Zotano, J., Olmedo-Cobo, J.A., Cunill-Artigas, R. \& Martínez-Ibarra, E., 2017. Descubrimiento y caracterización geográfica de una depresión ultramáfica en Sierra Bermeja: nuevos datos geomorfoedáficos, fitogeográficos y paleoecológicos. Pirineos, 172. http://dx.doi.org/10.3989/Pirineos.2017.172001

3. Gómez-Zotano, J., Cunill-Artigas, R., Olmedo-Cobo, J.A. \& Arias-García, J., 2018. Análisis pedoantracológico y propuesta de conectividad ecológica de Abies pinsapo en la Red Natura 2000 de Sierra Bermeja. In: Bosque mediterráneo y humedales: paisaje, evolución y conservación. Aportaciones desde la Biogeografia (eds. R.U. Gosálbez, M.C. Díaz, J.L. García, M.A. Serrano de la Cruz \& O. Jerez). Almud, Ediciones de Castilla-La Mancha, pp. 635-645, Ciudad Real.

4. Olmedo-Cobo, J.A., Cunill-Artigas, R., Martínez-Ibarra, E. y Gómez-Zotano, J. 2016. Nuevos datos paleoecológicos de Abies ssp. en el sur de España a partir del análisis pedoantracológico en Sierra Bermeja. In: Avances en Biogeografia: áreas de distribución: entre puentes y barreras (eds. J. Gómez, J. Arias, J.A. Olmedo \& J.L. Serrano). Editorial Universidad de Granada-Tundra Ediciones, pp. 581-581, Granada y Castellón.

5. Olmedo-Cobo, J.A., Cunill-Artigas, R., Martínez-Ibarra, E. \& Gómez-Zotano, J., 2017. Paleoecología de Abies sp. en Sierra Bermeja (sur de la península ibérica) durante el Holoceno medio a partir del análisis pedoantracológico. Bosque, 38(2): 259270. http://dx.doi.org/10.4067/S0717-92002017000200004

6. Olmedo-Cobo, J.A., Gómez-Zotano, J., Cunill-Artigas, R. \& Serrano-Montes, J.L. 2018. Controversia latifolias-coníferas sobre sustratos ultramáficos: una resolución desde las fuentes históricas y la pedoantracología. In: Bosque mediterráneo y humedales: paisaje, evolución y conservación. Aportaciones desde la Biogeografía (eds. R.U. Gosálbez, M.C. Díaz, J.L. García, M.A. Serrano de la Cruz \& O. Jerez). Almud, Ediciones de Castilla-La Mancha, pp. 242-253, Ciudad Real.

7. Olmedo-Cobo, J.A., Cunill-Artigas, R. \& Gómez-Zotano, J., 2019. The native status of Pinus pinaster on serpentine soils: charcoal analysis and palaeoenvironmental history in Sierra Bermeja (southern Iberian Peninsula, Spain). Vegetation History and Archaeobotany, 28: 417-432. https://doi.org/10.1007/s00334-018-0701-z

8. Olmedo-Cobo, J.A., Cunill-Artigas, R., Gómez-Zotano, J. \& Pardo-Martínez, R., 2019. Aportaciones del análisis pedoantracológico al conocimiento paleoecológico de Pinus pinaster en el sur de España: el caso de Sierra Bermeja. Boletín de la Asociación de Geógrafos Españoles, 80: 1-34. https://doi.org/10.21138/bage.2667

\begin{tabular}{|l|l|}
\hline Año de publicación & $2015(1), 2016(4), 2017$ (2 y 5), 2018 (3 y 6) y 2019 (7 y 8). \\
\hline Tipo de documento & Capítulos de libro (1, 3, 4 y 6) y artículos científicos $(2,5,7$ y 8). \\
\hline Importancia de la pedoantracología & $\begin{array}{l}\text { Alta: la pedoantracología constituye una de las metodologías de trabajo principales para la } \\
\text { consecución de los objetivos propuestos. }\end{array}$ \\
\hline Sondeos pedológicos & $\begin{array}{l}\text { 1 sondeo sobre litologías ígneas (1.364 m s. n. m.) (1, 2, 4 y 5). } \\
7 \text { sondeos sobre litologías ígneas (entre 638 y 1.364 m s. n. m.) (3 y 7). } \\
11 \text { sondeos sobre litologías ígneas (entre 146 y 1.364 m s. n. m.) (6 y 8). }\end{array}$ \\
\hline Objetivo/s principal/es & $\begin{array}{l}\text { Reconstruir la paleobiogeografía del pinsapo en el sur peninsular (1, 2, 3, 4 y 5). } \\
\text { Resolver la controversia científica latifolias-coníferas como vegetación climácica en } \\
\text { sustratos ultramáficos }(6,7 \text { y 8). }\end{array}$ \\
\hline Taxones identificados & $\begin{array}{l}\text { Especies: Arbutus unedo, Pinus pinaster. } \\
\text { Géneros: Abies, Cistus, Quercus. }\end{array}$ \\
\hline Valores de antracomasa & Entre 1,4 y 137.379 mg/kg. \\
\hline Dataciones radiocarbónicas & $\begin{array}{l}\text { No se realizan (1 y 2). } \\
10 \text { dataciones con cronologías comprendidas entre edades subactuales y 8.180 BP (3, 4, 5, 6 y 7). } \\
19 \text { dataciones con cronologías comprendidas entre edades subactuales y 8.180 BP. }\end{array}$ \\
\hline Tipo de aproximación & Geográfica. \\
\hline
\end{tabular}


Otros autores destacados son Olmedo y Gómez-Zotano, ambos del Departamento de Análisis Geográfico Regional y Geografía Física de la Universidad de Granada (UGR en adelante), y Pèlachs, del Departamento de Geografía de la UAB y miembro del GRAMP; en los tres casos su participación es del 41\%. Soriano, del Departamento de Geografía de la UAB y miembro del GRAMP, aparece en un $36 \%$ de las publicaciones, porcentaje que se reduce hasta el 32\% para Pérez-Obiol, del Departamento de Biología Animal, Biología Vegetal y Ecología (UAB).

El porcentaje se sitúa en un $18 \%$ para Bal, del Geode (Géographie de l'Environnement), en Toulouse-le-Mirail (Francia), coautora de 4 publicaciones. El mismo porcentaje en el caso de García-Codron, del Departamento de Geografía, Urbanismo y Ordenación del Territorio de la Universidad de Cantabria y miembro del Grupo de Investigación Estudio y Gestión del Medio Natural (GIMENA en adelante) y Martínez-Ibarra (Departamento de Análisis Geográfico Regional y Geografía Física de la UGR), reduciéndose hasta casi el 14\% para Carracedo (Departamento de Geografía, Urbanismo y Ordenación del Territorio de la Universidad de Cantabria y miembro del GIMENA).

Menor participación han tenido Nadal, Sánchez-Morales, Arias, Manel, García-Amorena y Beato, todos ellos con una significación en torno al $9 \%$.

Por último, los autores que aparecen citados una sola vez son Rubiales (Departamento de Sistemas y Recursos Naturales de la Universidad Politécnica de Madrid), Serrano (Departamento de Geografía Humana de la UGR), Pérez-Haase (Departamento de Botánica y Micología de la Universidad de Barcelona), Poblete (Departamento de Geografía de la Universidad de Oviedo), Pardo (Departamento de Análisis Geográfico Regional y Geografía Física de la UGR), Nunes (Departamento de Geografía de la UAB), Allée y García-Álvarez, ambos del Laboratorio de Geografía Física y Ambiental de la Universidad de Limoges.

\subsection{Fecha de publicación}

Los trabajos sobre pedoantracología en España se iniciaron en 2010 a partir de la defensa de la tesis doctoral de Cunill-Artigas: "Estudi interdisciplinari de l'evolució del límit superior del bosc durante el periode holocenic a la zona de Plaus de Boldís-Montarenyo, Pirineu central català. Pedoantracologia, palinologia, carbons sedimentaris i fonts documentals". No obstante, ya en 2008 la misma autora y colaboradores empezaron a trabajar con esta metodología, analizando las consecuencias en el paisaje de los nuevos usos del suelo en el límite superior del bosque (ver Cunill et al., 2008). A partir de entonces han sido publicados diferentes trabajos en los que esta disciplina tiene un peso sustancial en la investigación, ya sea como metodología principal o formando parte de una aproximación multidisciplinar.

Por fechas, 2017 y 2018 han sido los años más prolíficos en cuanto a publicaciones sobre pedoantracología se refiere, con un total de 9 publicaciones durante este bienio. Ello se debe, en buena medida, a determinados congresos y otros eventos de carácter científico en los que se divulga conocimiento $\mathrm{y}$, al mismo tiempo, existe un intercambio de información recíproco entre los diferentes grupos de trabajo. En este apartado destacan los Congresos de Biogeografía que, desde 2010 (Alicante) y hasta 2018 (Almagro, Ciudad Real), han permitido a los diferentes grupos de trabajo y, en especial al GRAMP como pionero en la aplicación de la pedoantracología en España-, estrechar lazos y colaborar con otras universidades y grupos de trabajo para favorecer el desarrollo de la disciplina.

\subsection{Revistas e idioma}

De las 22 publicaciones seleccionadas y analizadas, un total de 10 corresponden a artículos de revistas científicas, mientras que el resto se divide entre capítulos de libros (10) y tesis doctorales (2).

Las revistas en las que se han publicado estos ensayos son: Boletín de la Asociación Española de Geografía (BAGE), Bosque, Cuadernos Geográficos y Pirineos, en lo que respecta a publicaciones en castellano; y Quaternary International y Vegetation History and Archaeobotany, en habla inglesa.

Atendiendo a los indicadores de mayor impacto según el año de publicación, los trabajos sobre pedoantracología quedarían clasificados de la siguiente manera:

- Revistas indexadas en JCR (Journal Citation Reports): 4 de las 6 revistas quedarían incluidas en este índice (Boletín de la Asociación Española de Geografia, Bosque, Quaternary International y Vegetation History and Archaebotany). Los índices de impacto oscilan entre 0,409 (Bosque) y 2,482 (Vegetation History and Archaebotany) -años 2017 y 2019, respectivamente-, mientras que los cuartiles lo hacen entre el Q4 (Boletín de la Asociación Española de Geografía y Bosque) y el Q2 (Vegetation History and Archaebotany).

- Revistas indexadas en SJR (SCImago Journal Rank): el resto de revistas (Cuadernos Geográficos y Pirineos) quedan insertas en este índice, con factores de impacto que se situaban, respectivamente, en 0,216 y 0,274 durante el año 2017. Ambas revistas, por su parte, se incluyen en el Q3.

\section{4. Ámbito de trabajo}

Los primeros ensayos pedoantracológicos en España tuvieron lugar en la cara sur de la cordillera del Pirineo, a partir de los trabajos dirigidos por el GRAMP. Sin embargo, desde 2015 esta metodología empezó a aplicarse en otros sistemas montañosos españoles como la Cordillera Bética para, poco después, expandirse hacia el Sistema 
Central, la Cordillera Prelitoral Catalana y la Cordillera Cantábrica.

A día de hoy, y a pesar de que sigue siendo el norte de España donde se ha llevado a cabo un mayor número de trabajos de pedoantracología (54,5\%), los sistemas montañosos del sur del país - concretamente la Serranía de Ronda (Cordillera Bética) - también cuentan con una importante significación, concentrando casi el $41 \%$ del total. Por su parte, entre las zonas consideradas, es el Sistema Central el que muestra una representación más reducida $(4,5 \%)$, con tan solo una investigación en la Sierra de Gredos (ver García-Álvarez et al., 2017).

\subsection{Principales objetivos y líneas de aplicabilidad derivadas del análisis de la bibliografía consultada}

Tras la revisión pormenorizada de los objetivos de todas las publicaciones consultadas, se pueden establecer las siguientes categorías principales:

1. Contribuir al conocimiento acerca del límite altitudinal del bosque.

2. Mejorar el conocimiento biogeográfico (tanto presente como pasado) sobre una especie y/o especies concretas.

3. Determinar las causas (naturales y antrópicas) que han provocado cambios en los paisajes de montaña.

4. Conocer la historia de los incendios y la evolución de la dinámica del paisaje.

5. Ayudar a resolver controversias fitosociológicas clásicas en las que la comunidad científica se ha encontrado tradicionalmente dividida.

Por otro lado, las principales líneas de aplicabilidad derivadas del análisis de estos trabajos serían:

a. Consecución de información ecológica y paleoecológica a escala local.

b. Compresión, gestión y conservación de ecosistemas de montaña.

c. Planteamiento de modelos de conectividad ecológica.

d. Conocimiento de la historia de los incendios, tanto naturales como antrópicos.

e. Comparativa sobre la utilidad, ventajas e inconvenientes de diferentes fuentes a la hora de conseguir determinados objetivos.

f. Ayuda para la protección de una/s especie/s.

\subsection{Valores de antracomasa}

Los valores de antracomasa por puntos de muestreo arrojan cifras muy dispares según se consideren unos espacios u otros. Las cifras más bajas se sitúan en valores que oscilan entre los $0,2 \mathrm{mg} / \mathrm{kg}$ del Pirineo catalán (ver Cunill, 2010; Cunill et al., 2012, 2013) y los 1.373 mg/kg del Sistema Central (ver García-Álvarez et al., 2017), situándose la media de los valores más bajos de antracomasa en $626,27 \mathrm{mg} / \mathrm{kg}$. En el otro extremo, los valores más altos se sitúan entre los 28,77 y los $137.379 \mathrm{mg} / \mathrm{kg}$ de la Cordillera Cantábrica y Cordillera Bética, respectivamente. Por su parte, la media de los valores más altos de antracomasa se sitúa en $45.838,13 \mathrm{mg} / \mathrm{kg}$.

\subsection{Sondeos pedoantracológicos}

Dentro de este apartado se han analizado tanto el número de sondeos pedoantracológicos realizados como la litología sobre la cual se han llevado a cabo. En primer lugar, destaca la enorme variabilidad entre el número de sondeos de cada uno de los ensayos analizados. De esta forma encontramos trabajos en los que únicamente se ha llevado a cabo un solo muestreo pedológico, como por ejemplo los realizados en determinadas localizaciones del Pirineo catalán y la Cordillera Bética (ver Soriano et al., 2016; Olmedo-Cobo et al., 2017). En el primer caso, dicho sondeo forma parte de una aproximación multidisciplinar, adquiriendo de esta manera un carácter complementario. En segundo lugar, el número máximo de sondeos pedológicos realizados en un mismo ensayo se sitúa en 11 (ver Olmedo-Cobo et al., 2018, 2019a y b). La media, por su parte, quedaría en 5,375 sondeos por ensayo.

Respecto a la litología sobre la cual se han realizado los sondeos pedológicos, destaca sobremanera el gran peso de los materiales metamórficos $(57,1 \%)$ en el Pirineo catalán y Sistema Central (ver Cunill et al., 2008; García-Álvarez et al., 2017) e ígneos (42,9\%) en Sierra Bermeja (Cordillera Bética) (ver Gómez-Zotano et al., 2017; Olmedo-Cobo et al., 2017), representados por esquistos, gneises y filitas en el primer caso, y peridotitas en el segundo. Por su parte, los materiales sedimentarios fundamentalmente calizas- apenas representan un 14,3\% del total, restringiéndose únicamente a la Cordillera Cantábrica (ver Beato-Bergua et al., 2019).

\subsection{Dataciones radiocarbónicas}

El número de dataciones por ensayo ha oscilado entre 1 y 23 , situándose la media en 8,3 dataciones.

Las cronologías oscilan entre edades subactuales -apenas uno o dos siglos- y 10.796 BP. La media de las cronologías más recientes se sitúa en 1.206,13 BP, mientras que el promedio de las dataciones más antiguas -varias de ellas diez-milenarias- quedaría en 8.501,58 BP.

\subsection{Taxones identificados}

En este punto son las coníferas las que centran la atención de la mayoría de ensayos pedoantracológicos. Concretamente, el grueso de los trabajos gira en torno a especies como Abies alba o Pinus sylvestris/uncinata en el 
Pirineo catalán, Taxus baccata en la Cordillera Cantábrica o Abies pinsapo y Pinus pinaster en la Cordillera Bética. No obstante, algunas planifolias, como las del género Betula, también tienen un papel destacado, sobre todo en aquellas investigaciones cuyo objetivo es estudiar el límite superior del bosque o "treeline" a lo largo del Holoceno (ver Cunill, 2010; Cunill et al., 2012, 2013; GarcíaÁlvarez et al., 2017). También destacan otros géneros arbóreos como Corylus, Fagus, Populus y Quercus acompañados por Arbutus, Cistus, Juniperus, Ilex y familias como las leguminosas -especialmente en el Sistema Central-y, en menor medida, las ericáceas, fabáceas, y rosáceas.

\subsection{Principales dificultades encontradas durante el desarrollo de las investigaciones pedoantracológicas en España}

En este apartado aparece un denominador común: el gran porcentaje de carbones vitrificados y/o en mal estado que imposibilitó cualquier tipo de identificación taxonómica. Al mismo tiempo, durante el transcurso de las investigaciones analizadas se identificaron otras dificultades que se pueden resumir en los siguientes puntos:

- Características anatómicas específicas muy parecidas entre unas especies y otras, como por ejemplo, entre Pinus pinaster y Pinus halepensis, Abies alba y Abies pinsapo, y Pinus sylvestris y Pinus uncina$\mathrm{ta}$, siendo imposible discriminar el rango de especie en los dos últimos casos.

- Suelos que no se comportan como registros sedimentarios estratificados. Por tanto, cualquier muestra de carbón que pudiese ser objeto de interés para la investigación precisaría de una datación de forma independiente, con el consecuente coste económico.

- Valores de antracomasa reducidos y, por tanto, escasez de carbón. Si bien este hecho puede proporcionar información paleoecológica local importante referida al papel de los incendios a lo largo del tiempo en un determinado lugar-, al mismo tiempo supone un importante déficit de información, ya que la ausencia de registros (en forma de carbones) imposibilita que pueda llevarse a cabo identificación alguna.

- Tamaño muy reducido del carbón, lo que dificulta su tratamiento e identificación taxonómica en laboratorio, limitando en muchos casos su posterior datación radiocarbónica.

- Zonas de muestreo en localizaciones montañosas alejadas de cualquier vía de comunicación, ya sea asfaltada o no, lo que requiere de largas aproximaciones a pie por terrenos irregulares. Paralelamente, el porteo de material -tanto herramientas como muestras de suelo- supone un esfuerzo extra que precisa de la colaboración entre varias personas.
- Elevado coste de las dataciones radiocarbónicas, circunstancia que limita la elección de carbones con el fin de obtener cronologías que puedan ser de interés para la investigación en cuestión.

\subsection{Tipo de aproximación}

En este apartado aparecen dos vías fundamentales: geográfica y ecológica. En el primer caso, los sondeos pedológicos se establecen en diferentes localizaciones dentro de un extenso conjunto fisiográfico, permitiendo abarcar una amplia escala espacial. Por el contrario, en la aproximación ecológica los sondeos se concentran en una misma ladera, adquiriendo forma de transecto altitudinal.

En España es la aproximación geográfica la de mayor aplicación $(68,75 \%)$, siendo dominante en las investigaciones de la Cordillera Bética y Cordillera Cantábrica. Por su parte, la aproximación ecológica se circunscribe al Sistema Central y al Pirineo catalán $(31,25 \%)$, coincidiendo mayoritariamente con trabajos que estudian el límite superior del bosque.

\section{Discusión}

Cabe destacar que el volumen de información obtenido ha sido relativamente reducido, lo que se explica por el hecho de que la pedoantracología no ha sido una disciplina científica de larga e intensa tradición en España. Ello ha posibilitado que se pudiesen consultar de manera individualizada cada uno de los documentos con el fin de contrastar que los parámetros de búsqueda seleccionados habían generado bibliografía de interés para la presente investigación.

Esto difiere notablemente con lo que sucede en territorios de referencia como Francia, país pionero en la implantación de esta metodología a partir del trabajo de Thinon (1978). El país galo produce o colabora en gran parte de los ensayos pedoantracológicos realizados en Europa, tal y como se deduce de la síntesis de Nelle et al. (2013), realizada a partir del First International Workshop (2010), y posteriores (2013, 2017). En otros países europeos, como Italia, Alemania o República Checa también se ha desarrollado esta disciplina, si bien la producción bibliográfica en éstos es ostensiblemente más reducida que la producida por los autores franceses, donde destacan nombres como Brigitte Talon, Michel Tinon, Christopher Carcaillet y Marie-Claude Bal, entre otros.

En España, como ya se ha comentado, esta metodología fue implantada por Cunill (2010) durante el desarrollo de su tesis doctoral en el Pirineo Central catalán. No obstante, en Cunill et al. (2008) ya se hizo una primera aproximación pedoantracológica para analizar las consecuencias en el paisaje de los nuevos usos del suelo en el límite superior del bosque en el Pirineo.

Tras la aplicación pionera en la ladera sur del macizo pirenaico, los ensayos pedoantracológicos se fueron implementando paulatinamente en otros macizos montaño- 
sos del norte, centro y sur de España a lo largo de la pasada década, especialmente durante el bienio 2017-2018 (Figura 1). En el norte, son el GRAMP, de la UAB, y el GIMENA, de la Universidad de Cantabria (UC), los grupos que lideran las investigaciones de carácter pedoantra- cológico, si bien esta metodología forma parte de una aproximación interdisciplinar donde tienen cabida otras fuentes de información como los carbones sedimentarios o el análisis de metales pesados y polen, entre otras. En el caso del sur de España, es el grupo de investigación

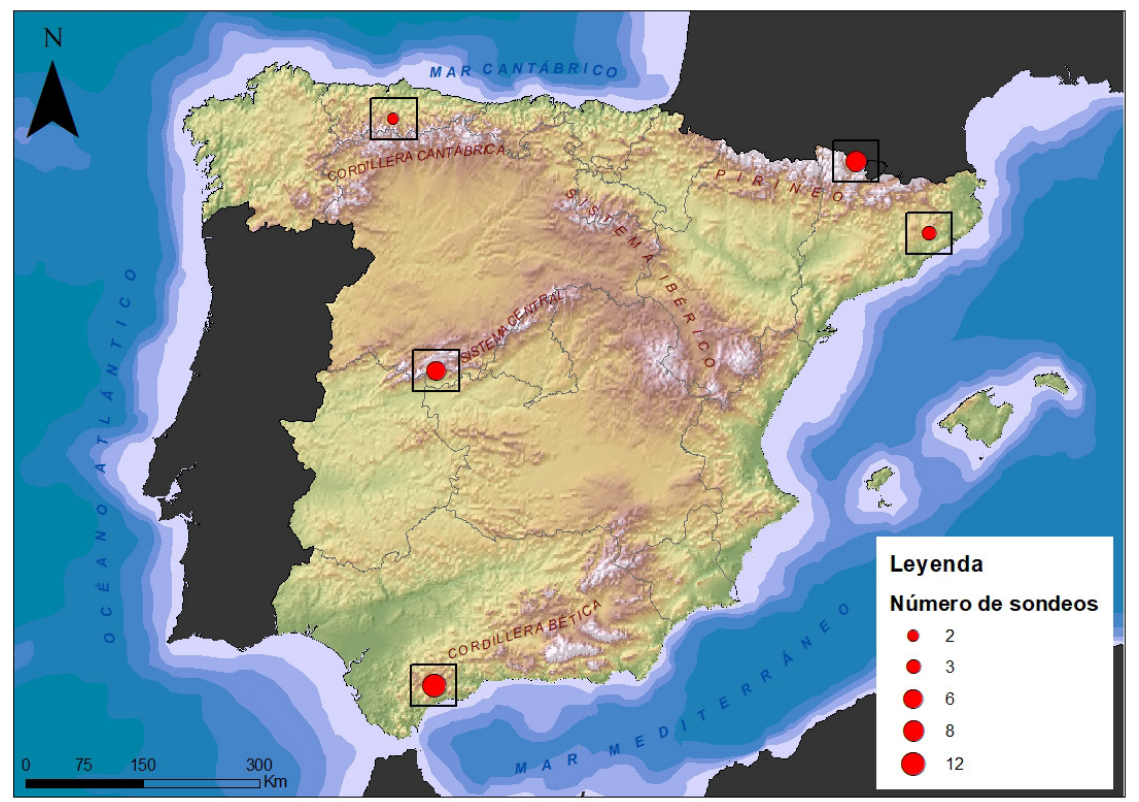

Figura 1: Número de sondeos pedoantracológicos según localización. Fuente: elaboración propia a partir de las capas de información del IECA.

Figure 1: Numbers of pedological excavations to according to location. Source: own elaboration from the information layers of the IECA.

PMAOT (Paisaje, Medio Ambiente y Ordenación Territorial en adelante), de la UGR, el que desarrolla el estudio pedoantracológico en el sector suroccidental de la Cordillera Bética, concretamente en el ámbito de la Serranía de Ronda (provincias de Málaga, Cádiz y Sevilla).

De la práctica totalidad de ensayos pedoantracológicos realizados en España dimanan algunas problemáticas comunes, como el alto coste de las dataciones radiocarbónicas - que limita el número de muestras que puedan enviarse a datar-, y el elevado porcentaje de carbones vitrificados. Este último fenómeno, cuya génesis no es aún conocida dentro del panorama científico (Valchalde et al., 2011), se suma a otros como el mal estado del carbón y/o la presencia de hongos. Todo ello, en conjunto, provoca que un porcentaje muy importante de los carbones seleccionados no pueda ser identificado taxonómicamente. Esto implica que pudieran sobreestimarse o subestimarse determinados taxones, lo que se traduciría en interpretaciones paleocológicas basadas en unas proporciones que podrían no corresponderse con las reales. Paralelamente, podrían haber quedado ocultos determinados taxones que pudiesen estar presentes en aquellos carbones no identificados, lo que conllevaría una interpretación incompleta a nivel paleocológico. Todo ello a pesar de los excepcionales valores de antracomasa que presentan algunos muestreos, como los llevados a cabo por García-Álvarez et al.
(2017) en la Sierra de Gredos, o los realizados por Gómez-Zotano et al. (2017) y Olmedo-Cobo et al. (2019a y b) en el contexto de la Serranía de Ronda, con cifras máximas que oscilan entre los 23.487 y $137.379 \mathrm{mg} / \mathrm{kg}$, respectivamente. No obstante, esta mayor riqueza de carbón no se tradujo necesariamente en el buen estado de conservación del mismo, ya que en muchos casos el porcentaje de carbones no identificados supuso más del 30\% del total de las muestras analizadas (ver García-Álvarez et al., 2017; Olmedo-Cobo et al., 2017).

Los altísimos valores de antracomasa anteriormente mencionados, que generalmente se restringen a altitudes medias y medias/altas (entre los 1.000 y $2.000 \mathrm{~m} \mathrm{s.} \mathrm{n.} \mathrm{m.)}$ en localizaciones silíceas (Pirineo catalán y Sistema Central) y peridotíticas (Sierra Bermeja, en la Cordillera Bética), contrastan con las exiguas cifras de antracomasa de sondeos realizados en cotas altas del Sistema Central (piso bioclimático oromediterráneo) y el Pirineo catalán (pisos bioclimáticos alpino y subalpino). Lo mismo sucede en enclaves de naturaleza carbonatada -calizas y dolomías-, como la Cordillera Cantábrica y algunos sectores de la Cordillera Bética, donde las cifras de antracomasa son muy reducidas.

Junto a ello hay que considerar el importante papel que ha desempeñado el fuego en la configuración del paisaje vegetal de la montaña mediterránea (Bond \& Keeley, 
2005). En este sentido, es poco probable que bajo estos condicionantes los reducidos valores de antracomasa en localizaciones sedimentarias concretas puedan deberse a la escasa incidencia del fuego -ya sea por causas naturales y/o antrópicas-, sino que obedezcan más bien a la escasa capacidad de las litologías calizas para retener el carbón. Esta posible correlación podría jugar un papel determinante a la hora de determinar futuras zonas potenciales para llevar a cabo investigaciones de carácter pedoantracológico. No obstante, la reducida lista de ensayos pedoantracológicos realizados hasta el momento sobre materiales sedimentarios en España obliga a seguir siendo prudentes a la hora de interpretar esta posible relación.

Sea como fuere, la presencia de carbón está directamente relacionada con las perturbaciones de incendios que podrían explicar directamente los cambios pasados en las comunidades (Whitlock \& Larsen, 2001). Es en este apartado donde la pedoantracología -como disciplina que se encarga de la cuantificación e identificación de carbones vegetales procedentes de suelos naturales-, ha tenido un papel muy significativo. Prueba de ello ha sido la incorporación de la disciplina como fuente complementaria para la reconstrucción de los incendios forestales, sobre todo en las investigaciones llevadas por los grupos de investigación que han trabajado en el norte de España (GRAMP y GIMENA). Desde el punto de vista de la Biogeografía, resulta esencial conocer tanto el combustible quemado como determinar la naturaleza de las perturbaciones (naturales o antrópicas) que ocasionaron aquellos incendios pretéritos (Pèlachs et al., 2016). En muchos casos, estos incendios constituyeron un factor prioritario en la génesis de determinados paisajes montanos, tal y como se deriva de los ensayos pedoantracológicos analizados en la presente investigación.

De igual forma, la pedoantracología se ha erigido como pieza clave para el estudio en los cambios del límite altitudinal del bosque o "treeline". Al igual que en el caso anterior -geohistoria de los incendios forestales-, también se constata una clara concentración de estos ensayos en determinados sistemas montañosos del centro y norte del país (aproximaciones ecológicas en el Sistema Central y el Pirineo catalán), mientras que ni en la Cordillera Bética ni en la Cordillera Cantábrica se ha llevado a cabo reconstrucción alguna del límite altitudinal del bosque a partir de la pedoantracología. Su aplicación en sistemas montañosos como Sierra Nevada podría suponer la consecución de una información paleoecológica esencial para comprender el comportamiento de estos ecotonos que han marcado el límite ecológico de la vegetación arbórea a lo largo de la historia. Las variaciones en dichos límites nos pueden informar de las respuestas que ofrecen las distintas especies a los diferentes pulsos climáticos acontecidos a lo largo del Holoceno y, en ocasiones, durante el pre-Holoceno. El conocimiento de estas dinámicas pasadas puede ofrecer información clave para predecir el comportamiento del límite superior del bosque, ayudando en la gestión de determinadas especies arbóreas. Todo ello en un contexto de Cambio Global, donde los espacios de alta montaña representan los ambientes más sensibles y vulnerables (Pèlachs et al., 2017).

Tal y como muestran los resultados, el horizonte temporal cubierto por la pedoantracología es muy amplio, abarcando prácticamente la totalidad del periodo Holoceno. Así lo avalan las distintas dataciones radiocárbonicas de varios carbones cuya antigüedad máxima es de 10.796 BP (ver Cunill et al., 2013), un alcance cronológico que según Carracedo et al., (2017) tan solo es igualado y/o superado por otras aproximaciones como la palinología y el estudio de carbones sedimentarios, a la hora de abordar la geohistoria de los incendios forestales.

El alcance de la pedoantracología también se extiende hacia el conocimiento paleobiogeográfico de especies arbóreas de especial interés ecológico. Tal es el caso de Abies pinsapo y Taxus baccata, coníferas que presentan actualmente una distribución menor que la que tuvieron durante los primeros albores del Holoceno, tras la última gran glaciación del Würm (Serra, 2009; Linares, 2011). En el caso del pinsapo (Abies pinsapo), los trabajos desarrollados por el grupo PMAOT de la UGR han revelado que este singular abeto, cuya distribución se encuentra actualmente muy reducida y fragmentada en el conjunto de la Serranía de Ronda (Cordillera Bética), presentó una extensión mayor durante el Holoceno. Así lo evidencian los registros de carbones milenarios de Abies sp. -muy probablemente Abies pinsapo- hallados en varios enclaves del macizo bético en los que este taxón está ausente en la actualidad. En este sentido, junto a la paleopoblación de Palmitera (Sierra Bermeja), habría que sumar el reciente hallazgo de carbón de Abies sp. -con cronologías milenarias- en la Sierra de Jaraestepar, núcleo central de la Serranía de Ronda que pudo actuar como corredor natural durante las rutas migratorias de esta especie en el Holoceno. Por su parte, la aplicación pionera del método pedoantracológico en la Sierra del Aramo (Cordillera Cantábrica) ha permitido constatar la presencia del tejo (Taxus baccata) durante, al menos, los últimos 3.450 años. La existencia de taxones arbóreos -como el tejo-en localizaciones actualmente deforestadas proporciona una información esencial para el conocimiento de la génesis de los actuales paisajes de montaña (ver Beato-Bergua et al., 2019).

Todo ello posibilita que se puedan obtener perspectivas diacrónicas detalladas a partir de las cuales se conozcan mejor los impactos antropogénicos. De esta forma, la determinación de la línea de base -escasa o nula influencia humana- de un determinado paisaje facilitará la comprensión sobre la distribución actual de la vegetación, pudiendo predecir posibles cambios futuros (Willis et al., 2007; Jackson \& Hobbes, 2009; Willis \& Bhagwat, 2010). Esto resulta esencial a la hora de favorecer una gestión forestal adecuada, sobre todo en un contexto de Cambio Global como el actual. Más aún cuando las decisiones se toman sobre especies relictas en peligro de extinción, como Abies pinsapo. Es por ello que la pedoantracología, junto con el resto de disciplinas paleoecológicas, puede ayudar de manera sustancial al conocimiento de estas dinámicas pretéritas, favoreciendo la 
compresión tanto del origen como de las diferentes perturbaciones que han generado los paisajes actuales de montaña.

\section{Conclusiones}

Tal y como queda reflejado en los diferentes ensayos pedoantracológicos analizados en la presente revisión, cada vez se apuesta de una manera más decidida por la colaboración entre las diferentes disciplinas de corte paleocológico. De una forma u otra, en todos los ensayos pedoantracológicos realizados en España tienen cabida aparte, lógicamente, de la pedoantracología-, otras metodologías paleoecológicas, si bien es en los trabajos realizados en el centro y norte de España donde esta complementariedad es más evidente, hecho que puede explicarse por la mayor abundancia de registros fósiles, al contrario de lo que ocurre en el tercio sur de España (López \& López, 1994). Estas aproximaciones -denominadas multiproxy-, donde la pedoantracología cada vez ocupa un lugar más destacado, resultan fundamentales para la consecución de los objetivos anteriormente mencionados, ya que las limitaciones de una metodología pueden ser compensadas por las potencialidades de otra. De esta forma, la pedoantracología ha mostrado algunas ventajas frente a otras disciplinas, como son su gran precisión espacial o su aplicación en enclaves en los que es muy difícil aplicar otras metodologías (Talon, 1998). Al mismo tiempo, y tal y como muestran las investigaciones pedoantracológicas realizadas en España y revisadas en este trabajo, son las coníferas las que suscitan buena parte del interés paleoecológico (Abies alba, Abies pinsapo, Taxus baccata y Pinus pinaster). Este hecho en sí mismo puede constituir una ventaja en el sentido de que éstas, por su propia caracterización anatómica, presentan una morfología estructural mucho más simple que la que poseen las angiospermas, que además son mucho más numerosas y diversas. Por tanto, el proceso de identificación taxonómica se agiliza notablemente, al tiempo que posibilita que puedan concretarse de una manera más precisa los taxones analizados.

Si bien la pedoantracología es una disciplina de reciente tradición en España, sobre todo si la comparamos con la bibliografía de países como Francia, su aplicación durante los últimos dos lustros se ha ido extendiendo de manera progresiva por diferentes sistemas montañosos, como el Pirineo catalán, la Cordillera Prelitoral catalana, la Cordillera Cantábrica, el Sistema Central y la Cordillera Bética.

De igual manera, queda garantizada su progresiva expansión hacia otros sistemas montañosos del país, como la Sierra de Gata (Sistema Central), así como hacia nuevas localizaciones del Macizo Asturiano (Cordillera Cantábrica) y Cordillera Bética (Sierra de Grazalema, Sierra del Pinar, Sierra de Líjar, Torcal de Antequera o Sierra de Camarolos, entre otras).

Las contribuciones derivadas de estos ensayos, en muchos casos, resultan esenciales a la hora de establecer estrategias de gestión adecuadas para determinadas coníferas, sobre todo cuando se consideraba a estas especies como introducidas - caso de Pinus pinaster en Sierra Bermeja-. Igualmente, y bajo el paraguas de la paleoecología, las perspectivas paisajísticas a largo plazo constituyen un mecanismo básico para poder comprender adecuadamente la génesis y evolución de determinados paisajes de montaña y, sobre ello, poder actuar eficientemente en la gestión de unos paisajes insertos en un contexto de Cambio Global.

No se trata de devolver a los ecosistemas sus condiciones de referencia, si no de conocer tanto su evolución como las diferentes perturbaciones que han ocasionado los paisajes presentes en la actualidad. El entendimiento de estas dinámicas a largo plazo debe constituir un pilar básico en la gestión de unos ecosistemas muy frágiles, como son los de la alta montaña, donde disciplinas como la pedoantracología -junto a otras aproximaciones palecológicas- han ofrecido testimonios muy precisos y útiles sobre la historia de determinados paisajes montanos en España.

\section{Agradecimientos}

Esta investigación ha sido financiada por una ayuda FPU (Formación de Profesorado Universitario) del Ministerio de Educación, Cultura y Deporte de España - (Ref. 18/03023), estando los resultados enmarcados en el proyecto denominado "Reconstrucción paleobiogeográfica de Abies pinsapo Clemente ex boiss. a partir del análisis pedoantracológico: nuevos datos para la gestión de la especie y su hábitat" (CSO2017-83576-P).

\section{Bibliografía}

Beato-Bergua, S., 2018. El patrimonio natural de la Sierra del Aramo (Montaña Central Asturiana) y la evolución de su paisaje. Tesis Doctoral, Universidad de Oviedo.

Beato-Bergua, S., 2019. El patrimonio natural de la Sierra del Aramo (Montaña Central Asturiana) y la evolución de su paisaje (Summary of Doctoral dissertation, Universidad de Oviedo, Spain). Boletín de la Asociación de Geógrafos Españoles, 80, 2756, 1-5.

Beato-Bergua, S., Poblete-Piedrabuena, M.A. \& Cunill-Artigas, R., 2019. Taxus baccata en la Sierra del Aramo (Macizo Central Asturiano). Boletín de la Asociación de Geógrafos Españoles, 81: 1-30. https://doi.org/10.21138/bage.2772

Bellot, F. \& Vieitez, E., 1945. Primeros análisis polínicos en las turberas galaicas. Anales del Instituto de Edafología, Ecología y Fisiología, 4: 281-307.

Bond, W.J. \& Keeley, J.E., 2005. Fire as a global "herbivore": the ecology and evolution of flammable ecosystems. Trends in Ecology and Evolution, 20: 387-394. https://doi. org/10.1016/j.tree.2005.04.025

Carcaillet, C. \& Talon, B., 1996. Aspects taphonomiques de la stratigraphie et de la datation de charbons de bois dans les sols: exemple de quelques sols des Alps. Géographie Physique et Quaternaire, 50(2): 233-244.

Carcaillet, C. \& Thinon, M., 1996. Pedoanthracological contribution to the study of the evolution of the upper treeline in 
the Maurienne valley (North French Alps): methodology and preliminary data. Review of Palaeobotany and Palynology, 91, 399-416. https://doi.org/10.1016/0034-6667(95)00060-7

Carracedo-Martín, V., Cunill-Artigas, R., García-Codrón, J.C., Pèlachs-Mañosa, A., Pérez-Obiol, R. \& Soriano-López, J.M., 2017. Fuentes para la geografía histórica de los incendios forestales. Algunas consideraciones metodológicas. Cuadernos Geográficos, 56(3): 66-89.

Cunill, R., 2010. Estudi interdisciplinari de l'evolució del límit superior del bosc durant el periodo holocènic a la zona de Plaus de Boldís-Montarenyo, Pirineu central català. Tesis Doctoral, Universidad Autónoma de Barcelona.

Cunill-Artigas, R., Pèlachs, A. \& Soriano, J.M., 2008. The consequences on landscape of new land uses in the upper forest line. In: Naturbanization: New identities and processes for ruralnatural areas (ed. M.J. Prados). CRC Press/Balkema, Leiden.

Cunill-Artigas, R., Soriano, J.M., Bal, M.C., Pèlachs, A. \& Pérez-Obiol, R., 2012. Holocene treeline changes on the south slope of the Pyrenees: a pedoanthracological analysis. Vegetation History and Archaeobotany, 21(4): 373-384. https:// doi.org/10.1007/s00334-011-0342-y

Cunill-Artigas, R., Soriano, J.M., Bal, M.C., Pèlachs, A., Rodríguez, J.M. \& Pérez-Obiol, R., 2013. Holocene high-altitude vegetation dynamics in the Pyrenees: a pedoanthracology contribution to an interdisciplinary approach. Quaternary International, 289: 60-70. https://doi.org/10.1016/j. quaint.2012.04.041

Cunill-Artigas, R., Pèlachs-Mañosa, A., Soriano, J.M., PérezObiol, R., Nadal-Tersa, J. \& Sánchez-Morales, M., 2018. Evolución paleobiogeográfica del abeto (Abies alba) en su límite meridional ibérico (Parc Natural del Montseny, Girona-Barcelona). In: Bosque mediterráneo y humedales: paisaje, evolución y conservación. Aportaciones desde la Biogeografía (eds. R.U. Gosálbez, M.C. Díaz, J.L. García, M.A. Serrano de la Cruz \& O. Jerez). Almud, Ediciones de Castilla-La Mancha, pp. 860-868, Ciudad Real.

Froyd, C.A. \& Willis, K.J., 2008. Emerging issues in biodiversity and conservation management: the need for a palaeoecological perspective. Quaternary Science Reviews, 27: 17231732. https://doi.org/10.1016/j.quascirev.2008.06.006

García-Álvarez, S., Bal, M.C., Allée, P., García-Amorena, I. \& Rubiales, J.M., 2017. Holocene treeline history of a highmountain landscape inferred from soil charcoal: The case of Sierra de Gredos (Iberian Central System, SW Europe). Quaternary International, 457(1): 85-98. https://doi. org/10.1016/j.quaint.2017.04.019

García-Codron, J.C., Pèlachs-Mañosa, A. \& Carracedo-Martín, V., 2016. Fuentes para la historia de los incendios forestales y su impacto en la vegetación: puentes y barreras metodológicas. In: Avances en Biogeografía: áreas de distribución: entre puentes y barreras (eds. J. Gómez, J. Arias, J.A. Olmedo \& J.L. Serrano). Editorial Universidad de GranadaTundra Ediciones, pp. 563-571, Granada y Castellón.

Gómez-Zotano, J., Olmedo-Cobo, J.A., Martínez-Ibarra, E. \& Cunill-Artigas, R., 2015. Descubrimiento y caracterización de una cuenca endorreica en la cumbre de Sierra Bermeja (provincia de Málaga). En Análisis espacial y representación geográfica: innovación y aplicación (eds. J. de la Riva, P. Ibarra, R. Montorio \& M. Rodrigues). Universidad de Zaragoza-AGE, pp. 1629-1638, Zaragoza.

Gómez-Zotano, J., Olmedo-Cobo, J.A., Cunill-Artigas, R. \& Martínez-Ibarra, E., 2017. Descubrimiento y caracterización geográfica de una depresión ultramáfica en Sierra Bermeja: nuevos datos geomorfoedáficos, fitogeográficos y paleoecológicos. Pirineos, 172. http://dx.doi.org/10.3989/ Pirineos.2017.172001

Gómez-Zotano, J., Cunill-Artigas, R., Olmedo-Cobo, J.A. \& Arias-García, J., 2018. Análisis pedoantracológico y propuesta de conectividad ecológica de Abies pinsapo en la Red Natura 2000 de Sierra Bermeja. In: Bosque mediterráneo y humedales: paisaje, evolución y conservación. Aportaciones desde la Biogeografía (eds. R.U. Gosálbez, M.C. Díaz, J.L. García, M.A. Serrano de la Cruz \& O. Jerez). Almud, Ediciones de Castilla-La Mancha, pp. 635-645, Ciudad Real.

Jackson, S.T. \& Hobbes, R.J., 2009. Ecological restoration in the light of ecological history. Science, 325: 567-568. https://doi.org/10.1126/science.1172977

Linares, J.C., 2011. Biogeography and evolution of Abies (Pinaceae) in the Mediterranean Basin. The roles of long-term climatic changes and glacial refugia. Journal of Biogeography, 38: 619-630. org/10.1111/j.1365-2699.2010.02458.x

López, P. \& López, J.A., 1994. Comparison of peats and archaeological samples in the Andalusian región, Spain. AASP Contributions Series, 29: 127-139.

Nelle, O., Talon, B. \& Robin, B., 2013. Pedoanthracology: Analysing soil charcoal to study Holocene palaeoenvironments. Quaternary International, 289: 1-4. https://doi. org/10.1016/j.quaint.2012.11.024

Oliva-Rodriguez Ariza, M., 2006. La antracología: métodos y objetivos. In: Arqueometría y arqueología medieval (ed. R. Carta). Universidad de Granada, Granada, pp.193-217, Granada.

Olmedo-Cobo, J.A., Cunill-Artigas, R., Martínez-Ibarra, E. \& Gómez-Zotano, J. 2016. Nuevos datos paleoecológicos de Abies ssp. en el sur de España a partir del análisis pedoantracológico en Sierra Bermeja. In: Avances en Biogeografía: áreas de distribución: entre puentes y barreras (eds. J. Gómez, J. Arias, J.A. Olmedo \& J.L. Serrano). Editorial Universidad de Granada-Tundra Ediciones, pp. 581-581, Granada y Castellón.

Olmedo-Cobo, J.A., Cunill-Artigas, R., Martínez-Ibarra, E. \& Gómez-Zotano, J., 2017. Paleoecología de Abies sp. en Sierra Bermeja (sur de la península ibérica) durante el Holoceno medio a partir del análisis pedoantracológico. Bosque, 38(2): 259-270. http://dx.doi.org/10.4067/ S0717-92002017000200004

Olmedo-Cobo, J.A., Gómez-Zotano, J., Cunill-Artigas, R. \& Serrano-Montes, J.L. 2018. Controversia latifolias-coníferas sobre sustratos ultramáficos: una resolución desde las fuentes históricas y la pedoantracología. In: Bosque mediterráneo y humedales: paisaje, evolución y conservación. Aportaciones desde la Biogeografía (eds. R.U. Gosálbez, M.C. Díaz, J.L. García, M.A. Serrano de la Cruz \& O. Jerez). Almud, Ediciones de Castilla-La Mancha, pp. 242-253, Ciudad Real.

Olmedo-Cobo, J.A., Cunill-Artigas, R. \& Gómez-Zotano, J., 2019. The native status of Pinus pinaster on serpentine soils: charcoal analysis and palaeoenvironmental history in Sierra Bermeja (southern Iberian Peninsula, Spain). Vegetation History and Archaeobotany, 28: 417-432. https://doi. org/10.1007/s00334-018-0701-z

Olmedo-Cobo, J.A., Cunill-Artigas, R., Gómez-Zotano, J. \& Pardo-Martínez, R., 2019. Aportaciones del análisis pedoantracológico al conocimiento paleoecológico de Pinus pinaster en el sur de España: el caso de Sierra Bermeja. Boletín de la Asociación de Geógrafos Españoles, 80: 1-34. https://doi.org/10.21138/bage.2667

Pèlachs, A., García-Codron, J.C., Soriano, J.M., Pérez-Obiol, R. \& Catalán, J., 2016. Papel de los incendios en las dinámicas forestales del Norte de la Península Ibérica durante el Holoceno. In: Avances en Biogeografia: áreas de distribución: entre puentes y barreras (eds. J. Gómez, J. Arias, J.A. Olmedo \& J.L. Serrano). Editorial Universidad de GranadaTundra Ediciones, pp. 553-562, Granada y Castellón. 
Pèlachs, A., Pérez-Obiol, R., Soriano, J.M., Cunill, R., Bal, M.C. \& García-Codron, J.C., 2017. The Role of Environmental Geohistory in High-Mountain Landscape Conservation. In: High Mountain Conservation in a Changing World. Advances in Global Change Research (eds. J. Catalan, J. Ninot \& M. Aniz). Springer, pp. 107-129, Berlin.

Saulnier-Talbot, É., 2016. Paleolimnology as a Tool to Achieve Environmental Sustainability in the Anthropocene: An Overview. Geosciences, 6(26): 1-11. https://doi. org/10.3390/geosciences6020026

Serra, L. 2009. 9580* Bosques mediterráneos de Taxus baccata (*). In: Bases ecológicas preliminares para la conservación de los tipos de hábitat de interés comunitario en España (eds. V.V.A.A). Ministerio de Medio Ambiente, y Medio Rural y Marino, Madrid.

Soriano, J.M., Pèlachs-Mañosa, A., Pérez-Obiol, R., Rodríguez, J.M. \& Cunill-Artigas, R., 2016. Dinámica del abeto en el Pirineo catalán durante los últimos 10.000 años. In: Avances en Biogeografía: áreas de distribución: entre puentes y barreras (eds. J. Gómez, J. Arias, J.A. Olmedo \& J.L. Serrano). Editorial Universidad de Granada-Tundra Ediciones, pp. 544-552, Granada y Castellón.

Talon, B., 1998. Evolution des zones supra-forestieres des Alpes sud-occidentales françaises au cours de l'Holocene analyse pedoanthracologique. Tesis Doctoral, Aix-Marseille Université.

Talon, B, Carcaillet, C. \& Thinon, M., 1998. Études pédoanthracologiques des variations de la limite supérieure des arbres au cours de 1'Holocene dans les Alps françaises. Géographie Physique et Quaternaire, 52: 195-208

Thinon, M., 1978. La pédoanthracologie: une nouvelle méthode d'analyse phytochronologique depuis le Néolithique (Pedoanthracology: a new method in phytochronological analysis since the Neolithic). Comptes Rendus de l'Académie des Sciences, Paris Serie D: Sciences Naturelles, 287: 1203-1206.

Thinon, M., 1992. L'analyse pédoanthracologique: aspects méthodologiques et applications. Tesis Doctoral. Université Paul Cézanne.

Turner, R., Roberts, N. \& Jones, M.D., 2008. Climatic pacing of Mediterranean fire histories from lake sedimentary microcharcoal. Global and Planetary Change, 63(4): 317 324. https://doi.org/10.1016/j.gloplacha.2008.07.002

Unger, D.F., 1846. Pflanzengeschichtliche Bemerkungen über den Kaiserwald bei Grätz. Botanische Zeitung, 7(17): 313-321.

Uzquiano, P., 1997. Antracología y métodos: implicaciones en la economía prehistórica, etnoarqueología y paleoecología. Trabajos de Prehistoria, 54(1): 145-154. https://doi. org/10.3989/tp.1997.v54.i1.383

Vargas-Jiménez, I., 2012. La entrevista en la investigación cualitativa: nuevas tendencias y retos. The interview in the qualitative research: trends and challengers. Revista Electrónica Calidad En La Educación Superior, 3(1): 119-139.

Vaschalde, C., Durand, A. \& Thiriot, J., 2011. Vitrification and craft fire in occidental Mediterranean. Describing characteristics, first results and research hypothesis. In: Saguntum: Papeles del Laboratorio de Arqueologia de Valencia. 5th International Meeting of Charcoal Analysis; The charcoal as cultural and biological heritage (eds. E. Badal, Y. Carrion, E. Grau, M. Macias \& M. Ntinou). Departament de Prehistòria i Arqueologia de la Universidad de Valencia, pp. 19-20, Valencia.

Willis, K.J. \& Birks, H.J.B., 2006. What is natural? The need for a long-term perspective in biodiversity conservation. Science, 314: 1261-1265. https://doi.org/10.1126/ science. 1122667

Willis, K.J., Araujo, M.B., Bennett, K.D., Figueroa-Rangel, B., Froyd, C.A. \& Myers, N., 2007. How can a knowledge of the past help to conserve the future? Biodiversity conservation and the relevance of long-term ecological studies. Philosophical Transactions of the Royal Society B: Biological Sciences, 362: 175-186. https://doi.org/10.1098/ rstb.2006.1977

Willis, K.J. \& Bhagwat, S.A., 2010. Questions of importance to the conservation of biological diversity: answers from the past. Climate of the Past, 6: 759-769. https://doi. org/10.5194/cp-6-759-2010

Whitlock, C. \& Larsen, C., 2001. Charcoal as a fire proxy. In: Tracking Environmental Change Using Lake Sediments. Volume 3: Terrestrial, Algal, and Siliceous Indicators (eds. J.P. Smol, H.J.B. Birks \& W.M. Last. Kluwer Academic, pp. 7597, Dordrecht. 